\title{
Genomic Characterization of Extended-Spectrum Cephalosporin-Resistant Salmonella enterica in the Colombian Poultry Chain
}

OPEN ACCESS

Edited by:

Marta Martins,

Trinity College, Dublin, Ireland

Reviewed by:

Min Yue,

Zhejiang University, China

Agnese Lupo,

Agence Nationale de Sécurité

Sanitaire de l'Alimentation, de

l'Environnement et du Travail (ANSES),

France

Patrick Rik Butaye,

Ross University School of Veterinary

Medicine, Saint Kitts and Nevis

${ }^{*}$ Correspondence: Joost Hordijk

j.hordijk@uu.n

tThese authors have contributed equally to this work

Specialty section:

This article was submitted to Antimicrobials, Resistance and Chemotherapy,

a section of the journal

Frontiers in Microbiology

Received: 11 June 2018 Accepted: 21 September 2018

Published: 26 October 2018

Citation:

Castellanos $L R$,

van der Graaf-van Bloois $L$,

Donado-Godoy P, León M, Clavijo V,

Arévalo A, Bernal JF, Mevius DJ,

Wagenaar JA, Zomer $A$ and Hordijk $J$

(2018) Genomic Characterization of

Extended-Spectrum

Cephalosporin-Resistant Salmonella enterica in the Colombian Poultry Chain. Front. Microbiol. 9:2431.

doi: 10.3389/fmicb.2018.02431
Luis Ricardo Castellanos ${ }^{1,2}$, Linda van der Graaf-van Bloois ${ }^{1}$, Pilar Donado-Godoy ${ }^{2}$, Maribel León ${ }^{3}$, Viviana Clavijo ${ }^{2,4}$, Alejandra Arévalo ${ }^{2}$, Johan F. Bernal ${ }^{2}$, Dik J. Mevius ${ }^{1,5}$, Jaap A. Wagenaar ${ }^{1,5}$, Aldert Zomer ${ }^{1 \dagger}$ and Joost Hordijk ${ }^{1 * t}$

${ }^{1}$ Department of Infectious Diseases and Immunology, Faculty of Veterinary Medicine, Utrecht University, Utrecht, Netherlands, ${ }^{2}$ Colombian Integrated Program for Antimicrobial Resistance Surveillance - Coipars, Corporación Colombiana de Investigación Agropecuaria - Corpoica, Mosquera, Colombia, ${ }^{3}$ Dirección Técnica de Inocuidad e Insumos Veterinarios, Instituto Colombiano Agropecuario - ICA, Bogotá, Colombia, ${ }^{4}$ Department of Biological Sciences, Los Andes University, Bogotá, Colombia, ${ }^{5}$ Department of Bacteriology and Epidemiology, Wageningen Bioveterinary Research, Lelystad, Netherlands

Salmonella enterica serovars have been isolated from Colombian broilers and broiler meat. The aim of this study was to investigate the diversity of ESBL/pAmpC genes in extended-spectrum cephalosporin resistant Salmonella enterica and the phylogeny of ESBL/pAmpC-carrying Salmonella using Whole Genome Sequencing (WGS). A total of 260 cefotaxime resistant Salmonella isolates, obtained between 2008 and 2013 from broiler farms, slaughterhouses and retail, were included. Isolates were screened by PCR for ESBL/pAmpC genes. Gene and plasmid subtyping and strain Multi Locus Sequence Typing was performed in silico for a selection of fully sequenced isolates. Core-genome-based analyses were performed per ST encountered. bla $\mathrm{CMY}_{\mathrm{M}-2-\text { like }}$ was carried in 168 isolates, 52 carried bla $\mathrm{CTX}_{-\mathrm{M}-2}$ group, 7 bla $\mathrm{SHV}_{\mathrm{SH}}, 5 \mathrm{a}$ combination

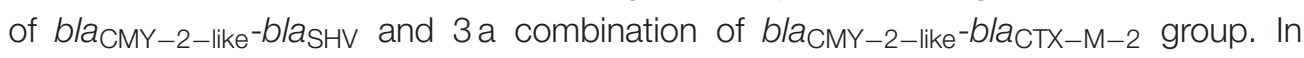
25 isolates no ESBL/pAmpC genes that were screened for were found. WGS characterization of 36 selected strains showed plasmid-encoded bla $\mathrm{CMY}_{-2}$ in 21 , bla $\mathrm{CTX}-\mathrm{M}-165$ in 11 and bla $\mathrm{SHV}_{\mathrm{SH}}$ in 7 strains. These genes were mostly carried on Incl1/ST12, IncQ1, and Incl1/ST231 plasmids, respectively. Finally, 17 strains belonged to $S$. Heidelberg ST15, 16 to S. Paratyphi B variant Java ST28, 1 to S. Enteritidis ST11, 1 to S. Kentucky ST152 and 1 to S. Albany ST292. Phylogenetic comparisons with publicly available genomes showed separate clustering of Colombian S. Heidelberg and S. Paratyphi B var. Java. In conclusion, resistance to extended-spectrum cephalosporins in Salmonella from Colombian poultry is mainly encoded by bla $\mathrm{CMY}_{2}$ and bla $\mathrm{CTX}_{-\mathrm{M}-165}$ genes. These genes are mostly associated with Incl1/ST12 and IncQ1 plasmids, respectively. Evolutionary divergence is observed between Colombian S. Heidelberg and S. Paratyphi B var. Java and those from other countries.

Keywords: Latin America, chicken, S. Paratyphi B d-tartrate positive, S. Heidelberg, S. Java, MLST, pMLST 


\section{INTRODUCTION}

Salmonella Enteritidis and S. Typhimurium have been reported as the most frequent serovars causing salmonellosis in humans worldwide (Hendriksen et al., 2011). According to data collected in the European Union (EU) in 2015 and the United States (USA) in 2013, S. Enteritidis accounted for 46 and $15 \%$ of Salmonella infections and S. Typhimurium for 16 and 13\%, respectively (Centers for Disease Control and Prevention (CDC), 2016; European Food Safety Authority (EFSA) and European Centre for Disease Prevention and Control (ECDC), 2016). Likewise, $S$. Typhimurium and $S$. Enteritidis are the most isolated serovars from human cases in Colombia. Their overall prevalence among human isolates between 2005 and 2011 was 32 and 28\%, respectively (Rodríguez et al., 2017).

Among foods of animal origin, poultry products (e.g., eggs) have been primarily associated with $S$. Enteritidis infections in humans while $S$. Typhimurium infections are associated with a wider range of sources including pork, beef and poultry products (Mughini-Gras et al., 2014; Antunes et al., 2016). Nevertheless, in broilers and chicken meat the prevalence of serotypes other than $S$. Enteritidis and $S$. Typhimurium have been on the rise over the last 2 decades (van Pelt et al., 2003; Foley et al., 2011; Wagenaar et al., 2013). Baseline studies of Salmonella performed between 2005 and 2006 in broiler chickens in the EU showed $S$. Infantis, $S$. Mbandaka, and $S$. Hadar to be highly prevalent (European Food Safety Authority (EFSA), 2007). In the year 2006, S. Infantis, S. Enteritidis and S. Paratyphi B dtartrate positive (here referred as $S$. Paratyphi B variant Java) were the most frequent serovars in broiler meat in the EU (European Food Safety Authority (EFSA), 2007). More recently, $S$. Infantis, $S$. Enteritidis, $S$. Mbandaka, and $S$. Ohio were the most prevalent serovars in broilers and broiler meat in 2015 (European Food Safety Authority (EFSA) and European Centre for Disease Prevention and Control (ECDC), 2016). In North America, S. Kentucky, S. Enteritidis, S. Typhimurium, S. Heidelberg, and $S$. Infantis are reported as the most prevalent serovars in broilers and ground chicken meat in the USA (United States Department of Agriculture (USDA) - Food Safety and Inspection Service (FSIS), 2013) while in Canada, S. Heidelberg, S. Kentucky and $S$. Enteritidis are the most prevalent in broilers and broiler meat (Public Health Agency of Canada, 2016). In Colombia, baseline studies performed by the Colombian integrated program for antimicrobial resistance surveillance (Coipars), demonstrated $S$. Paratyphi B var. Java, and $S$. Heidelberg to be the most prevalent serovars in broiler farms and meat at retail. Serovar distribution at farm level was 76 and 23\%, respectively (DonadoGodoy et al., 2012b), and at the retail level 45 and 19\%, respectively (Donado-Godoy et al., 2014). A similar distribution of $S$. Paratyphi B var. Java and $S$. Heidelberg, has been reported in chicken meat at retail in Guatemala (35 and 16\% respectively) (Jarquin et al., 2015) and broilers at slaughter in Venezuela (62 and 31\% respectively) (Boscán-Duque et al., 2007).

Isolates of S. Paratyphi B var. Java and S. Heidelberg are often multi drug resistant (Denny et al., 2007; Dutil et al., 2010; Antunes et al., 2016; Liakopoulos et al., 2016) and have been associated with carriage of plasmid-mediated extended spectrum $\beta$-lactamases (ESBLs) and plasmid associated AmpC $\beta$-Lactamases in poultry (pAmpC) (Antunes et al., 2016). For instance, $S$. Paratyphi B var. Java isolates from poultry in Europe have been found carrying the ESBL gene bla $a_{\mathrm{CTX}-\mathrm{M}-1}$ and the pAmpC gene bla $a_{\mathrm{CMY}-2}$ (Doublet et al., 2014; Mevius et al., 2015; Veldman et al., 2016). In turn, S. Heidelberg has been associated to bla $a_{\mathrm{CMY}-2}$ in North America (Andrysiak et al., 2008; Folster et al., 2012) and poultry products imported from South America into Europe (Liakopoulos et al., 2016). In South America $S$. Heidelberg has been associated with bla $a_{\mathrm{CTX}-\mathrm{M}-2}$ (Antunes et al., 2016). To date, no data of the genetic determinants of Extended Spectrum Cephalosporin (ESC) resistance in Salmonella from broilers and chicken meat in Colombia are available. These data are highly relevant to understand the epidemic spread of ESBL/pAmpC-producing Salmonella in poultry resulting in frequent occurrence in chicken meat in multiple countries.

Serotyping has traditionally been used for the epidemiological investigation of Salmonella, but does not provide information about the evolutionary relatedness of strains. Sequence based methodologies such as Multi Locus Sequence Typing (MLST) and Whole Genome Sequencing (WGS) have been proposed as a replacement of serotyping to identify evolutionary and epidemiological relatedness (Achtman et al., 2012; Ashton et al., 2016; Nadon et al., 2017). Additionally, information on the genetic basis of antimicrobial resistance (AMR) and plasmids harboring AMR genes can be readily obtained from WGS assemblies (Zankari et al., 2012; Carattoli et al., 2014). Altogether, the objectives of this study were to investigate the diversity of ESBL/pAmpC genes and encoding plasmids found in ESCresistant $S$. enterica from broilers and broiler meat in Colombia and to determine the genetic relatedness with Salmonella strains from other countries using MLST and core-genome alignments.

\section{MATERIALS AND METHODS \\ Isolates of Salmonella enterica}

The isolates included in this study originated from different cross-sectional baseline studies conducted between 2008 and 2013 at different stages during the development of Coipars. In these studies, non-clinical samples were obtained from three different levels of poultry production in Colombia: Broiler farms (Donado-Godoy et al., 2012b), broilers at slaughter (DonadoGodoy et al., 2015b) and broiler meat at retail (DonadoGodoy et al., 2012a, 2014, 2015a). The methodology for sampling, random isolation (i.e., without antimicrobials during enrichment) of Salmonella and antimicrobial susceptibility testing with the BD Phoenix automated system, was previously described in detail for the studies in broiler farms, broilers at slaughter and broiler meat at retail mentioned above. Previous results of the prevalence of Salmonella and resistance to ESC at the different levels of poultry production, are summarized in Table 1.

For the present study, all available $S$. enterica isolates $(n=673)$ were considered. The 673 isolates belonged to 578 production flocks, 28 flocks from broiler farms, 140 from slaughterhouses and 410 from retail (Table 1). Next, ESC-resistant isolates 
TABLE 1 | Prevalence and origin of Salmonella isolates used in this study, distribution of ESBL/pAmpC genes and selection of isolates for WGS.

\begin{tabular}{|c|c|c|c|c|c|c|c|c|}
\hline & $\begin{array}{c}\text { Number of } \\
\text { samples }\end{array}$ & $\begin{array}{l}\text { Salmonella- } \\
\text { positive } \\
(\%)\end{array}$ & $\begin{array}{c}\text { ESBL/pAmpC- } \\
\text { producing } \\
\text { Salmonella (\%) }\end{array}$ & $b_{\text {laMY-2-like }}$ & $b / a_{\mathrm{CTX}-\mathrm{M}-2}$ group & $b a_{\mathrm{SHV}}$ family & $\begin{array}{c}\text { bla }_{\mathrm{CMY}-2-l i k e}- \\
\text { bla }_{\mathrm{CTX}-\mathrm{M}-2} \\
\text { group }\end{array}$ & $\begin{array}{c}\text { bla }_{\mathrm{CMY}-2-l i k e}- \\
\text { bla } \\
\text { family }\end{array}$ \\
\hline Farms & 74 & $28(38)$ & $17(61)$ & $16 / 2^{b}$ & - & - & - & $1 / 1^{b}$ \\
\hline 2008 & 65 & 23 & 17 & 16 & - & - & - & 1 \\
\hline 2009 & 9 & 5 & - & - & - & - & - & - \\
\hline Slaughter & 644 & $140(22)$ & 82 (59) & $54 / 4^{b}$ & $25 / 2^{b}$ & - & $2 / 2^{b}$ & $1 / 1^{b}$ \\
\hline 2008 & 40 & 5 & 3 & 3 & - & - & - & - \\
\hline 2012 & 251 & 76 & 39 & 31 & 8 & - & - & - \\
\hline 2013 & 353 & 59 & 40 & 20 & 17 & - & 2 & 1 \\
\hline Retail & 1554 & $410(26)$ & $136(33)$ & $98 / 7^{\mathrm{b}}$ & $27 / 6^{b}$ & $7 / 7^{b}$ & $1 / 1^{b}$ & $3 / 3^{b}$ \\
\hline 2009 & 179 & 38 & 11 & 11 & - & - & - & - \\
\hline 2010 & 387 & 156 & 35 & 29 & 1 & 4 & - & 1 \\
\hline 2011 & 823 & 170 & 76 & 49 & 22 & 2 & 1 & 2 \\
\hline 2012 & 165 & 46 & 14 & 9 & 4 & 1 & - & - \\
\hline Total & 2272 & $578(25)$ & $235(41)$ & $168 / 13^{b}$ & $52 / 8^{b}$ & $7 / 7^{b}$ & $3 / 3^{b}$ & $5 / 5^{b}$ \\
\hline
\end{tabular}

a Based on PCR screening of ESBL/PAmpC genes.

${ }^{b} E S B L / p A m p C$-positive samples/Selected for whole genome sequencing.

of Salmonella were selected based on suspected phenotypic resistance to cefotaxime, as previously measured with the $\mathrm{BD}$ Phoenix (Donado-Godoy et al., 2012b) and interpreted using the CLSI 2014 clinical breakpoints for Enterobacteriaceae (MIC $\geq 4 \mu \mathrm{g} / \mathrm{ml}$ ) (Clinical and Laboratory Standards Institute (CLSI), 2014). For all flocks with multiple ESC-resistant isolates, each first isolate was selected to make sure we included epidemiologically unrelated isolates only.

As a result, a total of 260 isolates were selected for ESCresistance characterization. The selected isolates from farms originated from drag swabs of fecal material from the floor of broiler houses and fresh feces directly from the chicken. These isolates originated from 19 broiler farms $(n=19)$ (DonadoGodoy et al., 2012b). Isolates at slaughter, originated from cecal content and carcass rinse from 32 slaughterhouses $(n=84)$ (Donado-Godoy et al., 2015b). At retail, isolates originated from carcass rinse from 143 retail suppliers (Donado-Godoy et al., 2012a, 2014) and chicken thighs meat from 8 retail suppliers ( $n=157$ ) (Donado-Godoy et al., 2015a). The isolates originated from 18 out of 32 departments (i.e. provinces) of Colombia. Together, these 18 departments are responsible for more than $90 \%$ of the chicken population in the country. A map of Colombia with the location of these departments is available in Supplementary Figure 1.

Information related to the origin of the samples and the prevalence of Salmonella is shown in Table 1.

\section{PCR Screening of ESBL/pAmpC Genes}

For the present investigation, the 260 isolates selected as mentioned above, were screened by PCR for the presence of $\mathrm{ESBL} / \mathrm{pAmpC}$ genes families using previously described primers for $b l a_{\mathrm{TEM}}$ and bla $a_{\mathrm{SHV}}$ families, bla $a_{\mathrm{CMY}-2-\text { like }}$ and $b l a_{\mathrm{CTX}-\mathrm{M}}$ family (Dierikx et al., 2010); bla $a_{\mathrm{CTX}-\mathrm{M}-1}$ group (Carattoli et al., 2008); bla $_{\mathrm{CTX}-\mathrm{M}-2}$ group (Jiang et al., 2006); bla $_{\mathrm{CTX}-\mathrm{M}-8}$ group (Hopkins et al., 2006); bla $\mathrm{CTX- \textrm {M } - 9}$ group (Paauw et al., 2006); $b l a_{\mathrm{OXA}-1-\mathrm{like}}, b l a_{\mathrm{OXA}-2-\mathrm{like}}$ and $b l a_{\mathrm{OXA}-10-\text { like }}$ (Voets et al., 2011).

\section{Selection of Strains and WGS}

Based on the diversity of gene families found after PCR screening, a representative selection of isolates was made and further subjected to WGS. Due to its high prevalence, a random selection was made using the Random function in Microsoft ${ }^{\circledR}$ Excel to assign random numbers to isolates positive for $b l a_{\mathrm{CMY}-2-\text { like }}$ and $b l a_{\mathrm{CTX}-\mathrm{M}-2}$ group. The size of selection was fixed to the square root of the number of resulting positive strains for these genes. This resulted in $13 b b_{\mathrm{CMY}-2-l i k e}{ }^{-}$and 8 bla $a_{\mathrm{CTX}-\mathrm{M}-2}$ group-carrying isolates. In addition and due to their low prevalence, all positive isolates for bla $a_{\mathrm{SHV}}$ family and combinations of $b l a_{\mathrm{CMY}-2-\text { like }}-b l a_{\mathrm{SHV}}$ family and $b l a_{\mathrm{CMY}-2-\mathrm{like}}{ }^{-}$ $b l a_{\mathrm{CTX}-\mathrm{M}-2}$ group were included. An overview of the selection of isolates is shown in Table $\mathbf{1 .}$

Isolation of genomic DNA from selected isolates was performed using the UltraClean ${ }^{\circledR}$ Microbial DNA Isolation Kit (Mo Bio-Qiagen, USA). WGS was performed on Illumina MiSeq and NextSeq platforms (Illumina, USA) using $2 \times 250$-bp reads and $2 \times 150-$ bp reads, respectively. Genomes were assembled with SPAdes v3.10.1 (Bankevich et al., 2012).

\section{In silico Characterization of ESBL/pAmpC Gene Variants}

Subtyping of ESBL/pAmpC gene variants was performed using ResFinder 2.1 (Zankari et al., 2012). Investigation of resistance genes with an identity percentage $<100 \%$ was done using BLAST 2.6.0+ (Camacho et al., 2009).

\section{In silico Characterization of ESBL/pAmpC-Carrying Plasmids}

Plasmid content of selected strains was investigated using PlasmidFinder 1.3 and pMLST 1.4 (Carattoli et al., 2014). 
Identification of plasmids associated to the ESBL/pAmpC genes was based on co-localization on the same contig as resulted from ResFinder and PlasmidFinder analysis. Since this was not possible for all genomes, transformation of plasmids harboring each of the ESBL/pAmpC variants identified with ResFinder was performed together with selective culturing as described before (Castellanos et al., 2017). This was done to obtain the plasmid types identified with PlasmidFinder in transformed Escherichia coli $\mathrm{DH} 10 \mathrm{~B}$ harboring the different ESBL/pAmpC variants identified. Afterwards, the sequences of the transformed plasmids were used as reference to map against the genomes of the selected strains. To this purpose, transformants were sequenced with Illumina MiSeq and NextSeq sequencing as described above. Chromosomal contigs in transformants were detected and removed by mapping against the E. coli $\mathrm{DH} 10 \mathrm{~B}$ genome sequence (GenBank accession number: CP000948.1) using BLAST. The remaining contigs were considered to be part of the ESBL/pAmpC carrying plasmid and were used as a reference. Next, the contigs of the initially sequenced selected strains were aligned to the obtained reference plasmid sequences using MUSCLE (Edgar, 2004). Alignments were made on selected strains according to the ESBL/pAmpC gene variants they harbored. Resulting aligned contigs were selected and considered as newly inferred ESBL/pAmpC-carrying plasmids.

\section{In silico MLST and Serotype Prediction}

To determine the population structure of the selected ESCresistant Salmonella, 7-gene MLST at the strain level was performed in silico with MLST 1.8 (Larsen et al., 2012). Serotype was predicted using the Salmonella In Silico Typing Resource (SISTR) (Yoshida et al., 2016). Whole genome phylogenetic analyses were performed for S. Heidelberg ST15 and S. Paratyphi B var. Java ST28. Given the limited number of isolates $(n=1)$, this was not performed for S. Enteritidis ST11, S. Kentucky ST152 or S. Albany ST292.

\section{Collection of Publicly Available Genomes for Phylogenetic Comparisons}

Genome sequences of $S$. Paratyphi B var. Java ST28, were downloaded from Enterobase ${ }^{1}$ (last accessed: 12-Sept-2017) for comparison. Likewise, sequences of S. Heidelberg ST15 were obtained from Enterobase ${ }^{1}$ (last accessed: 11-Jan-2017). Only genomes with data available for their year of isolation, country of origin and source were considered for S. Heidelberg ST15. For both S. Paratyphi B var. Java ST28 and S. Heidelberg ST15, the genomes were collected disregarding their susceptibility to $3^{\text {rd }}$ generation cephalosporin's or to other antimicrobials. Additionally, the quality of genomes obtained in this study and the downloaded genomes was assessed with CheckM (Parks et al., 2015). Only genomes with $>98 \%$ completeness score, when compared against the set of genomic markers for S. enterica, were included. MLST designation was amended using a custom BLAST-based tool ${ }^{2}$.

\footnotetext{
${ }^{1}$ https://enterobase.warwick.ac.uk/

${ }^{2}$ https://github.com/tseemann/mlst
}

\section{Core Genome Phylogenetic Analysis}

Whole genome analysis was performed by a core-genome alignment using Parsnp v1.2 (Treangen et al., 2014). Recombination regions in the core genome alignment were detected and filtered using Gubbins (Croucher et al., 2015). Phylogenetic maximum likelihood (mid-point rooted) trees were constructed with the recombination-filtered core genomes alignments using FastTree $2^{3}$ (Price et al., 2010) and visualized with FigTree ${ }^{4}$.

\section{Data Availability}

The obtained genome sequences of the Salmonella strains selected for WGS (Table 2) and those of the transformed E. coli $\mathrm{DH} 10 \mathrm{~B}$ strain with the ESBL/pAmpC-carrying plasmids used as reference have been deposited in the short-read archive of the ENA under Project Number: PRJEB23610.

\section{RESULTS}

\section{PCR Screening of ESBL/pAmpC Genes}

After PCR screening of the 260 isolates, 235 isolates were positive for the genes screened for. 168 were positive for $b l a_{\mathrm{CMY}-2-\text { like }}, 52$ for $b l a_{\mathrm{CTX}-\mathrm{M}-2}$ group, 7 for bla $a_{\mathrm{SHV}}$ family, 5 harbored a combination of $b l a_{\mathrm{CMY}-2-\mathrm{like}}-b l a_{\mathrm{SHV}}$ family and 3 a combination of bla $a_{\mathrm{CMY}-2-\text { like }}-b l a_{\mathrm{CTX}-\mathrm{M}-2}$ group. In 48 isolates, bla $a_{\mathrm{TEM}}$ was co-located with $b l a_{\mathrm{CMY}-2-\text { like, }} b l a_{\mathrm{CTX}-\mathrm{M}-2}$ group or bla $a_{\mathrm{SHV}}$. In 25 isolates no ESBL/pAmpC genes that were screened for were encountered.

The distribution of positive samples for Salmonella according to their source, year and ESBL/pAmpC genes is shown in Table $\mathbf{1}$.

\section{Selection of Strains for WGS}

After PCR characterization, a random selection of $b l a_{\mathrm{CMY}-2-\text { like }}$ positive isolates $(n=13)$ and $b a_{\mathrm{CTX}-\mathrm{M}-2}$ group $(n=8)$ together with all positive isolates for bla $a_{\mathrm{SHV}}$ family $(n=7), b l a_{\mathrm{CMY}-2-\text { like }^{-}}$ bla $_{\mathrm{SHV}}$ family $(n=5)$ and $b l a_{\mathrm{CMY}-2-\text { like }}-b l a_{\mathrm{CTX}-\mathrm{M}-2}$ group $(n=3)$ were included for WGS. In total, 36 isolates were selected and subjected to WGS. The list of selected isolates and the results of the characterization of ESBL/pAmpC genes, plasmid types, serotypes, and strain MLST based on WGS is shown in Table 2.

\section{Characterization of ESBL/pAmpC Gene Variants}

After WGS characterization of resistance genes, 13 strains harbored $b l a_{\mathrm{CMY}-2}, 8$ bla $a_{\mathrm{CTX}-\mathrm{M}-165}, 6$ bla $a_{\mathrm{SHV}-12}$ and 1 bla $a_{\mathrm{SH}-129}$. Additionally, three harbored the combination of $b l a_{\mathrm{CMY}-2}-b l a_{\mathrm{CTX}-\mathrm{M}-165}$, four $b l a_{\mathrm{CMY}-2}-b l a_{\mathrm{SHV}-129}$ and

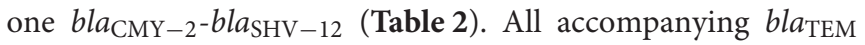

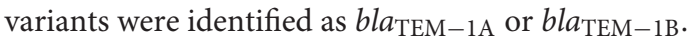

\section{In silico Characterization of ESBL/pAmpC-Carrying Plasmids}

Co-localization of ESBL/pAmpC and plasmid replicon genes in the same contig was observed for 17 out of 36 strains selected for

\footnotetext{
${ }^{3}$ http://meta.microbesonline.org/fasttree/

${ }^{4}$ http://tree.bio.ed.ac.uk/software/figtree/
} 
TABLE 2 | Origin of the Salmonella isolates selected for WGS and results of the characterization of their ESBL/pAmpC genes, plasmids, and strains.

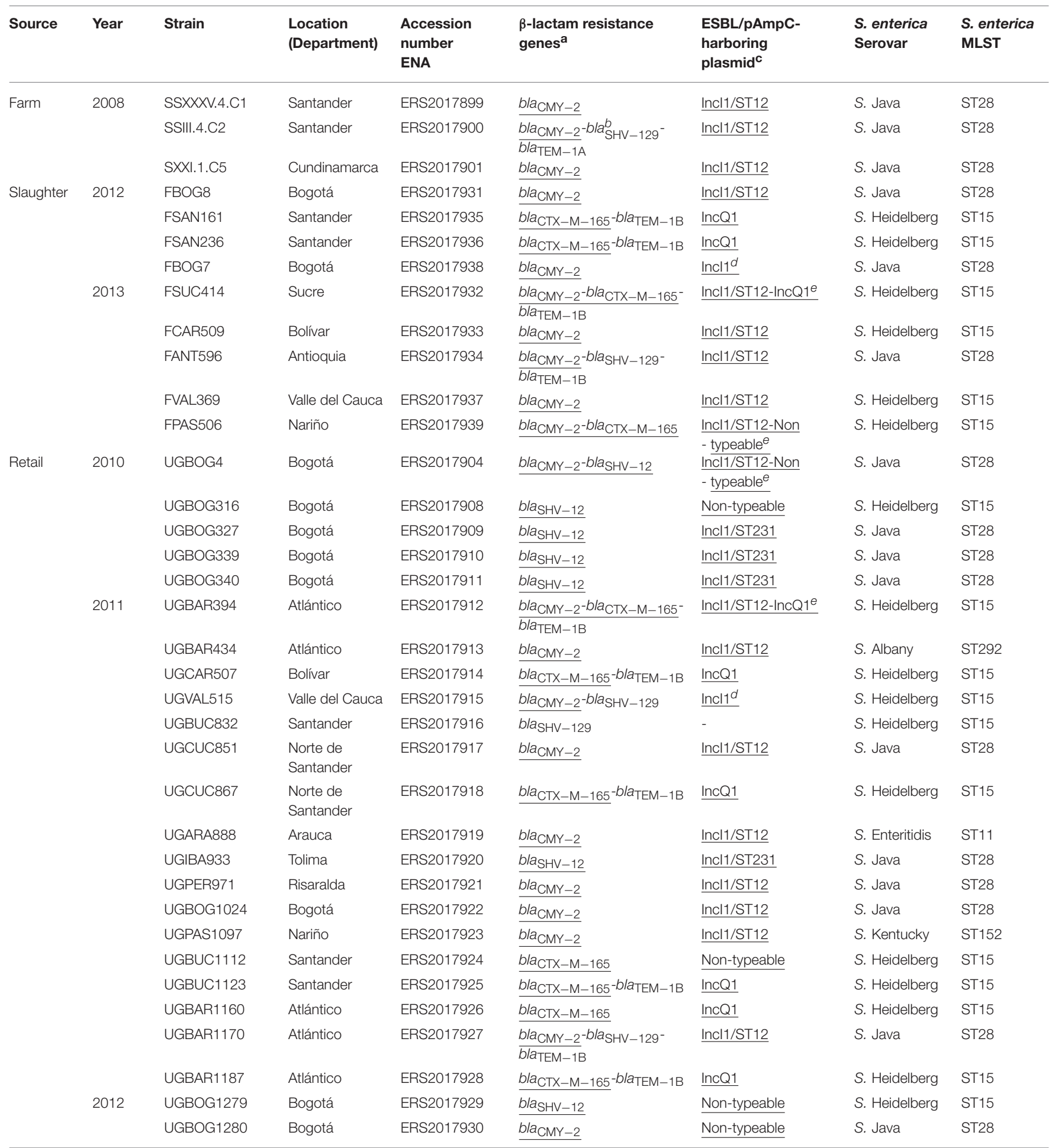

a The genes present in the contigs selected for plasmid characterization have been underlined.

${ }^{b}$ blaSHV-129 was non-transferable after electroporation experiments.

${ }^{c}$ The plasmids carrying the $\beta$-lactam resistance genes have been underlined.

${ }^{d}$ These plasmids missed one allele from the PMLST scheme.

e Characterization of the two ESBL/PAmpC genes was performed in separate plasmid contigs. 
WGS. For the remaining strains, co-localization was determined by analyzing selected contigs harboring ESBL/pAmpC and plasmid replicon genes using MUSCLE. Genes conferring resistance to other antimicrobials only co-localized with

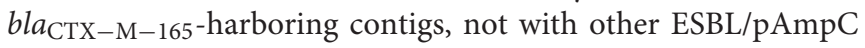
genes (Supplementary Table 1). In detail, one transformant was obtained carrying either a bla $a_{\mathrm{CMY}-2^{-}}$, bla $a_{\mathrm{CTX}-\mathrm{M}-165^{-}}$ or bla $a_{\mathrm{SHV}-12}$-harboring plasmid. The plasmids isolated from strains UGBOG4 (bla $\left.a_{\mathrm{CMY}-2}\right)$, UGBAR1160 (bla $\left.a_{\mathrm{CTX}-\mathrm{M}-165}\right)$ and UGBOG327 (bla $a_{\mathrm{SHV}-12}$ ) (Supplementary Table 1) were used as a reference to map against the genomes of all selected strains. The bla $a_{\text {SHV-129 }}$ gene present in five selected strains was not transferable by transformation from any of the strains, suggesting chromosomal localization of this gene.

After characterizing the plasmid contigs of the selected

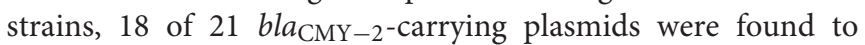
belong to IncI1/ST12, 1 was non-typeable based on the PCR Based Replicon Typing (PBRT) scheme used in PlasmidFinder (Carattoli et al., 2014). Two were designated IncI1, but the plasmid from strain UGVAL515 lacked the pilL allele and the plasmid from FBOG7 lacked the sogS allele. Nonetheless, these two plasmids remained single-allele variants of IncI1/ST12. For bla $a_{\mathrm{CTX}-\mathrm{M}-165}, 9$ of 11 plasmids harbored the IncQ1 plasmidreplicon and 2 remained non-typeable. Four of seven $b l a_{\mathrm{SHV}-12}$ plasmids belonged to IncI1/ST231 and three remained nontypeable (Table 2 and Supplementary Table 1).

\section{Strain MLST, Serotype Characterization, and Core Genome Phylogeny}

After using 7-gene MLST and the Salmonella In Silico Typing Resource (SISTR), 17 strains belonged to S. Heidelberg ST15, 16 to $S$. Paratyphi B var. Java ST28, 1 to $S$. Enteritidis ST11, 1 to $S$. Kentucky ST152 and 1 to S. Albany ST292 (Table 2). Further, whole genome analysis was performed for ST28 and ST15 isolates. For the phylogenetic analysis, additional genomes for ST28 $(n=60)$ and ST15 $(n=1221)$ were selected from Enterobase.warwick.ac.uk disregarding their characteristics of susceptibility to 3rd generation cephalosporins and used to construct the phylogenetic maximum likelihood trees. All Colombian genomes belonging to ST28 and ST15 formed a single cluster in the phylogenetic analysis. Phylogenetic trees for ST28 and ST15 with data regarding the source, year and country of the strains, are shown in Figures 1 and 2 respectively. No clustering related to the presence or absence of an ESBL/pAmpC gene is observed in Figure 1, suggesting the observed clustering is related to the geographical origin of S. Paratyphi B var. Java ST28 strains and not to the presence of an AMR gene. Likewise, in Figure 2, a cluster of $S$. Heidelberg ST15 strains originating from Colombian poultry is observed. Furthermore, the Colombian strains from ST28 and ST15 were disseminated in multiple departments within the country. A map of Colombia with the location of origin of ST28 and ST15 isolates selected for WGS is available in Supplementary Figure 2. An additional table with the metadata of strains selected for the construction of the ST28 and ST15 phylogenies is available as Supplementary Table 2 .

\section{DISCUSSION}

In summary, bla $a_{\mathrm{CMY}-2}, \quad b l a_{\mathrm{CTX}-\mathrm{M}-165}, \quad b l a_{\mathrm{SHV}-12}$, and bla $a_{\mathrm{SHV}-129}$ are described as the most prevalent ESBL/pAmpC genes conferring resistance to ESC in $S$. enterica isolated from the Colombian poultry chain between 2008 and 2013. According to the objectives of the present study, the collection of isolates served to reflect maximum diversity of ESBL/pAmpC genes in different years, departments and levels of the poultry production chain in Colombia.

The finding of bla $a_{\mathrm{CMY}-2}$ as the main cause of ESC resistance is comparable to a previous report of ESBL/pAmpC-producing E. coli in Colombian poultry. In that study, it was encountered a prominent association of this gene with IncI1/ST12 plasmids (Castellanos et al., 2017). Those results suggested occurrence of horizontal gene transfer of this plasmid lineage between heterogeneous $E$. coli STs. The results from the present study,

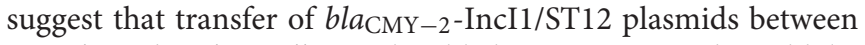
E. coli and Salmonella is also likely to occur, and could be considered a driver of the frequent occurrence of this resistance gene along the poultry chain. This particular gene-plasmid association has been described in E. coli from Brazil (da Silva et al., 2017), Salmonella from the USA (Folster et al., 2010) and E. coli and Salmonella from Europe (Accogli et al., 2013; Smith et al., 2015), suggesting an epidemiological link between

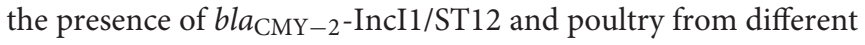
countries. Nevertheless, WGS of these plasmids is necessary to assess the level of genetic relatedness among them and estimate the potential transmission of these plasmids between E. coli and Salmonella.

After PCR screening, bla $a_{\mathrm{CTX}-\mathrm{M}-2}$ group was found to be the most prevalent ESBL gene among Colombian isolates. After subtyping a selection of these isolates with WGS, these genes

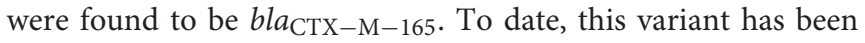
solely reported in an isolate of Klebsiella pneumoniae from a urine sample in Chile and reported in 2016 (Accession number: KP727572) without further epidemiological records. Noticeably,

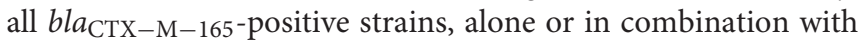
other bla genes, were identified in S. Heidelberg ST15. In our collection of isolates comprising the years 2008 to 2013, bla $a_{\mathrm{CTX}-\mathrm{M}-165}$ is only detected from the year 2010 onwards (Table 1). Not taking into account the potential bias that could occur by having isolates comprising a period of time no longer than 5 years, this finding may suggest a recent introduction of this gene in Colombian poultry, and until 2013, is limited to S. Heidelberg ST15. However, analysis of recently collected isolates of S. enterica and other Enterobacteriaceae is necessary to confirm this hypothesis.

After electroporation experiments, $b l a_{\mathrm{SHV}-129}$ remained nontransferable and no plasmid markers were identified in its harboring contigs, which ranged in size between 2100 and $8594 \mathrm{bp}$. Therefore, it is likely that this gene is chromosomally located. Furthermore, the gene was found in two different serovars, S. Paratyphi B var. Java ST28, and S. Heidelberg ST15. It can be hypothesized that its transfer could be associated to an integrative or transposable element. Initial screening of transposases and Insertion Sequences (IS) using BLAST 


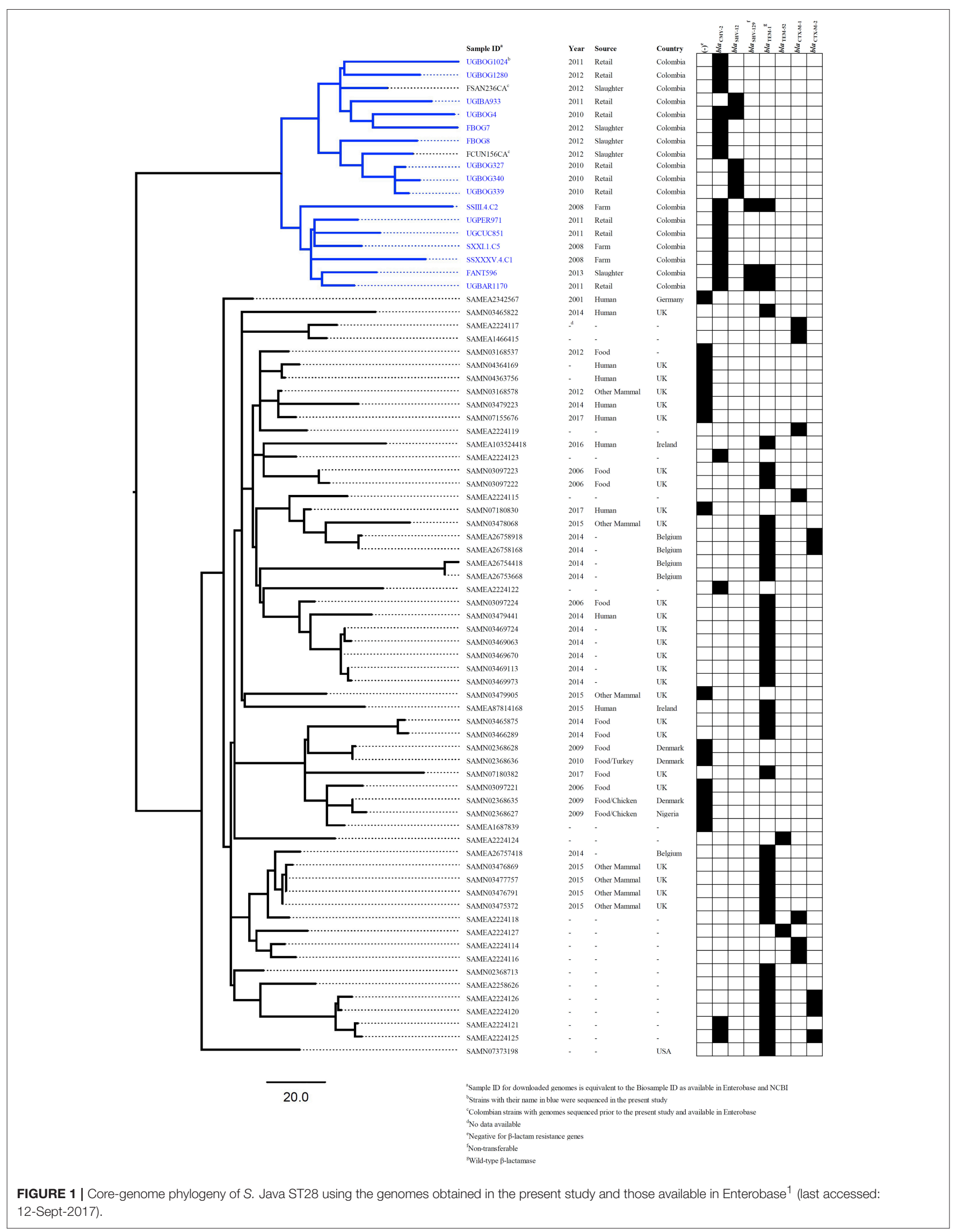




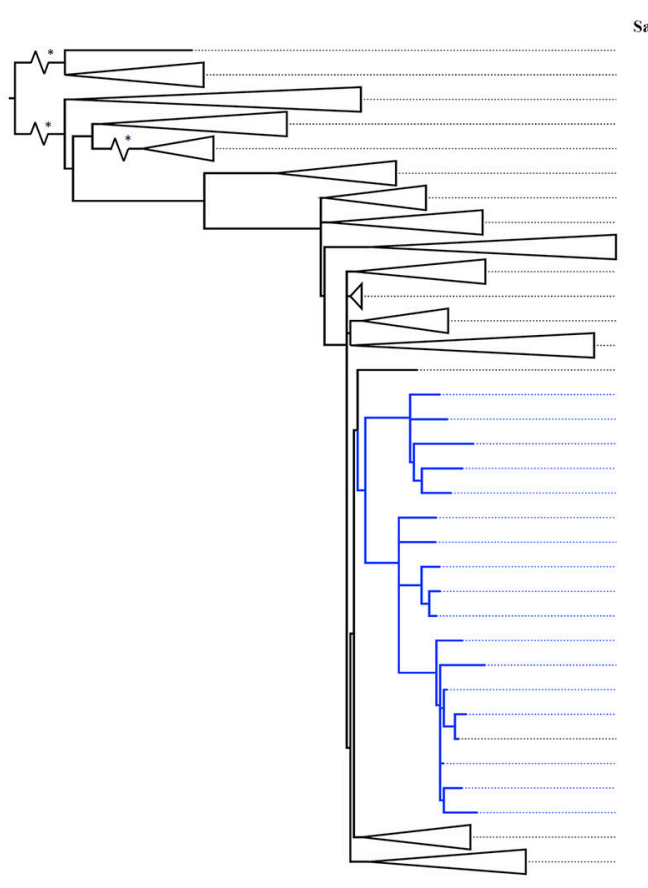

\begin{tabular}{|c|}
\hline $\begin{array}{l}\text { Sample ID }{ }^{\mathrm{a} / \text { Number of strains }} \\
\text { SAMN02843731 }\end{array}$ \\
\hline 8 \\
\hline 13 \\
\hline 4 \\
\hline 6 \\
\hline 13 \\
\hline 2 \\
\hline 53 \\
\hline 366 \\
\hline 74 \\
\hline 4 \\
\hline 20 \\
\hline 186 \\
\hline$\underline{\text { SAMN03476166 }}$ \\
\hline$\underline{\mathrm{UGBOG}} 316^{\mathrm{C}}$ \\
\hline$\overline{\text { UGVAL } 515}$ \\
\hline UGBUC8 32 \\
\hline FVAL369 \\
\hline UGBOG1279 \\
\hline UGCUC867 \\
\hline UGBAR 1160 \\
\hline$\underline{\text { FPAS506 }}$ \\
\hline UGBUC1123 \\
\hline$\overline{\text { UGBUC1112 }}$ \\
\hline UGCAR507 \\
\hline FSUC414 \\
\hline FSAN161 \\
\hline FSAN236 \\
\hline SAMN03842020 $^{\mathrm{d}}$ \\
\hline$\underline{\text { UGBAR394 }}$ \\
\hline$\overline{\text { UGBAR } 1187}$ \\
\hline$\underline{\text { FCAR509 }}$ \\
\hline 99 \\
\hline 370 \\
\hline
\end{tabular}

Years of isolation
2002
$2002-2016$
$1961-2015$
$2014-2015$
$2012-2014$
$2009-2016$
$2007-2012$
$2003-2015$
$1998-2016$
$1997-2015$
$2006^{6}$
$2003-2007$
$2002-2016$
2014
2010
2011
2011
2013
2012
2011
2011
2013
2011
2011
2011
2013
2012
2012
2013
2011
2011
2013
$2004-2016$
$2000-2016$

Source of isolation (\%)
Shellfish
Human $(37 \%)$
Human $(69 \%)$
Macadamia nuts $(75 \%)$
Human $(83 \%)$
Bovine $(85 \%)$
Human $(100 \%)$
Poultry $(92 \%)$
Poultry $(87 \%)$
Poultry $(85 \%)$
Bovine $(100 \%)$
Human $(45 \%) ;$ Manure $(45 \%)$
Poultry $(84 \%)$
Human
Poultry
Poultry
Poultry
Poultry
Poultry
Poultry
Poultry
Poultry
Poultry
Poultry
Poultry
Poultry
Poultry
Poultry
Poultry
Poultry
Poultry
Poultry
Poultry $(97 \%)$
Poultry $(83 \%)$

Country of origin (\%)
Thailand
UK $(50 \%)$
UK $(61 \%)$
USA (75\%)
UK $(83 \%)$
USA (100\%)
France (50\%); Canada (50\%)
USA (100\%)
USA (99\%)
USA $(85 \%)$
USA (100\%)
USA (95\%)
USA ( $99 \%)$
UK
Colombia
Colombia
Colombia
Colombia
Colombia
Colombia
Colombia
Colombia
Colombia
Colombia
Colombia
Colombia
Colombia
Colombia
Colombia
Colombia
Colombia
Colombia
USA ( $99 \%)$
USA $(99 \%)$

*Lenght of the branches have been reduced 10 -fold for display purposes

${ }^{\text {a }}$ ample ID for downloaded genomes is equivalent to the Biosample D as available in Enterobase and NCBI

${ }^{b}$ Name of indivdual strains have been underlined

'Strains with their name in blue colour were sequenced in the present study

${ }^{\mathrm{d}}$ Colombian strain with genomes sequenced prior to the present study and available in Enterobase

${ }^{\mathrm{e}} \mathrm{A}$ range of years is provided for multiple strains

${ }^{\mathrm{f}}$ Multiple strains originated in the same year

${ }^{8}$ The main Source and main Country of origin of multiple strains is presented as percentage in brackets (\%)

FIGURE 2 | Core-genome phylogeny of S. Heidelberg ST15 using genomes obtained in this study and those with complete metadata selected from Enterobase ${ }^{1}$ (last accessed: 11-Jan-2017).

(Siguier, 2006) on the contigs harboring these genes detected several IS families flanking the sequences inside the contigs. Nevertheless, given the restricted size of the contigs no definite association with a unique IS element (e.g., AMR-associated IS) was possible. In such a case, complementing the short-read WGS data with additional data obtained through long-read sequencing is necessary to confirm the chromosomal location of this gene and its association to a particular mobile genetic element. This approach could also be used for further characterization of ESBL/AmpC-plasmids that were non-typeable according to the PBRT scheme used in PlasmidFinder, which could have also been affected by the limitations of genome and plasmid assembly of short-reads.

As mentioned previously, ESC and non-ESC $S$. Paratyphi $B$ var. Java and $S$. Heidelberg were reported as the most prevalent serovars in the Colombian poultry chain (DonadoGodoy et al., 2012b, 2014). In the present study, investigation of resistance to cefotaxime showed a total of 235 (41\%) resistant isolates from which, 17 (61\%) originated from farms, $82(59 \%)$ from slaughterhouses and 136 (33\%) from retail (Table 1). Noteworthy, the prevalence of resistance diminishes from one level of production to the other. From previous studies, $S$. Paratyphi B var. Java and $S$. Heidelberg were the most prevalent serovars encountered at the farm level (Donado-Godoy et al., 2012b) and a larger diversity of serovars was found at retail, with more than 10 different serovars isolated repeatedly (DonadoGodoy et al., 2014). As observed in the present study, strains belonging to $S$. Paratyphi B var. Java and $S$. Heidelberg had a higher prevalence of ESC-resistance in comparison to the other serovars. These results indicate that the higher prevalence of $S$. Paratyphi B var. Java and $S$. Heidelberg, accounted in large part for the higher prevalence of ESC-resistance at the farm level and the presence of different serovars resulted in the reduction of resistance along the production chain, which is reflected in the lower prevalence of ESC-resistance at retail.

As anticipated, the analysis of Salmonella strains using MLST in addition to the resolution provided by WGS data has proven to be very useful in showing clustering of Colombian strains belonging to $S$. Paratyphi B var. Java ST28. According to the phylogenetic analysis including ESBL/pAmpC-positive and-negative strains (Figure 1), the clustering seems to occur independently of ESC-susceptibility and may be related to the geographical origin of the strains. Whether the cluster of Colombian S. Paratyphi B var. Java ST28 represents a particular separate lineage circulating in the country, or is present elsewhere 
in Latin America is a question that requires further investigation. At the time of publication of the present study no genomes of ST28 from other Latin American countries were publicly available and the comparisons within the region were limited to the strains we sequenced and analyzed.

In conclusion, resistance to ESC in S. enterica from Colombian poultry is mainly caused by $b l a_{\mathrm{CMY}-2}$ and $b l a_{\mathrm{CTX}-\mathrm{M}-165}$ genes. These genes are mostly associated with IncI1/ST12 and IncQ1 plasmids, respectively. The resolution provided by WGS was appropriate to assess the evolutionary divergence of strains from Colombian poultry belonging to S. Paratyphi B var. Java ST28. Dissemination of ESBL/pAmpC genes in Salmonella is mainly due to the carriage of plasmids encoding these genes in strains belonging to S. Paratyphi B var. Java ST28 and S. Heidelberg ST15.

\section{AUTHOR CONTRIBUTIONS}

JW, PD-G, JH, AZ, DM, LG-vB, and LC contributed to the design of the study. LG-vB and AZ performed the formal analysis. JW Contributed funding acquisition. LG-vB, PD-G, ML, VC, $\mathrm{AA}, \mathrm{JB}, \mathrm{AZ}$, and LC conducted material and data collection. JW, $\mathrm{PD}-\mathrm{G}$, and AZ provided biological, laboratory and computational

\section{REFERENCES}

Accogli, M., Fortini, D., Giufrè, M., Graziani, C., Dolejska, M., Carattoli, A., et al. (2013). IncI1 plasmids associated with the spread of CMY-2, CTX-M-1 and SHV-12 in Escherichia coli of animal and human origin. Clin. Microbiol. Infect. 19:E238-E240. doi: 10.1111/1469-0691.12128

Achtman, M., Wain, J., Weill, F. X., Nair, S., Zhou, Z., Sangal, V., et al. (2012). Multilocus sequence typing as a replacement for serotyping in Salmonella enterica. PLoS Pathog. 8:e1002776. doi: 10.1371/journal.ppat.10 02776

Andrysiak, A. K., Olson, A. B., Tracz, D. M., Dore, K., Irwin, R., Ng, L. K., et al. (2008). Genetic characterization of clinical and agri-food isolates of multi drug resistant Salmonella enterica serovar Heidelberg from Canada. BMC Microbiol. 8:89. doi: 10.1186/1471-2180-8-89

Antunes, P., Mourão, J., Campos, J., and Peixe, L. (2016). Salmonellosis: the role of poultry meat. Clin. Microbiol. Infect. 22, 110-121. doi: 10.1016/j.cmi.2015.12.004

Ashton, P. M., Nair, S., Peters, T. M., Bale, J. A., Powell, D. G., Painset, A., et al. (2016). Identification of Salmonella for public health surveillance using whole genome sequencing. PeerJ 4:e1752. doi: 10.7717/peerj.1752

Bankevich, A., Nurk, S., Antipov, D., Gurevich, A. A., Dvorkin, M., Kulikov, A. S., et al. (2012). SPAdes: a new genome assembly algorithm and its applications to single-cell sequencing. J. Comput. Biol. 19, 455-477. doi: $10.1089 / \mathrm{cmb} .2012 .0021$

Boscán-Duque, L. A., Arzálluz-Fisher, A. M., Ugarte, C., Sánchez, D., Wittum, T. E., and Hoet, A. E. (2007). Reduced susceptibility to quinolones among Salmonella serotypes isolated from poultry at slaughter in Venezuela. J. Food Prot. 70, 2030-2035. doi: 10.4315/0362-028X-70.9.2030

Camacho, C., Coulouris, G., Avagyan, V., Ma, N., Papadopoulos, J., Bealer, K., et al. (2009). BLAST+: architecture and applications. BMC Bioinformatics 10:421. doi: 10.1186/1471-2105-10-421

Carattoli, A., García-Fernández, A., Varesi, P., Fortini, D., Gerardi, S., Penni, A., et al. (2008). Molecular epidemiology of Escherichia coli producing extendedspectrum $\beta$-lactamases isolated in Rome, Italy. J. Clin. Microbiol. 46, 103-108. doi: 10.1128/JCM.01542-07

Carattoli, A., Zankari, E., García-Fernández, A., Larsen, M. V., Lund, O., Villa, L., et al. (2014). In Silico detection and typing of plasmids using PlasmidFinder resources. JH, JW, DM, and AZ supervised the study. LC wrote the original draft. JH, JW, DM, LG-vB, and AZ critically reviewed the manuscript.

\section{FUNDING}

This project was financed by internal funding of Utrecht University, the Netherlands.

\section{ACKNOWLEDGMENTS}

The authors wish to thank Birgitta Duim, Arjen Timmerman, and Mirlin Spaninks from Utrecht University for assistance in obtaining the WGS of strains and Maria Fernanda Valencia from Corpoica for technical assistance. WHO-AGISAR is acknowledged for facilitating the exchange of researchers and knowledge between research groups.

\section{SUPPLEMENTARY MATERIAL}

The Supplementary Material for this article can be found online at: https://www.frontiersin.org/articles/10.3389/fmicb. 2018.02431/full\#supplementary-material and plasmid multilocus sequence typing. Antimicrob. Agents Chemother. 58, 3895-3903. doi: 10.1128/AAC.02412-14

Castellanos, L. R., Donado-Godoy, P., León, M., Clavijo, V., Arevalo, A., Bernal, J. F., et al. (2017). High heterogeneity of Escherichia coli sequence types harbouring ESBL/AmpC genes on IncI1 plasmids in the Colombian poultry chain. PLoS ONE 12:e0170777. doi: 10.1371/journal.pone.0170777

Centers for Disease Control and Prevention (CDC). (2016). Centers for Disease Control and Prevention National Salmonella Surveillance Annual Report, 2013. 1-89. Available online at: https://www.cdc.gov/nationalsurveillance/pdfs/ salmonella-annual-report-2013-508c.pdf

Clinical and Laboratory Standards Institute (CLSI). (2014). Clinical and Laboratory Standards Institute M100-S24. Performance Standards for Antimicrobial Susceptibility Testing. 24th Informational Supplement. Wayne, PA: Clinical and Laboratory Standards Institute.

Croucher, N. J., Page, A. J., Connor, T. R., Delaney, A. J., Keane, J. A., Bentley, S. D., et al. (2015). Rapid phylogenetic analysis of large samples of recombinant bacterial whole genome sequences using Gubbins. Nucleic Acids Res. 43:e15. doi: 10.1093/nar/gku1196

da Silva, K. C., Cunha, M. P. V., Cerdeira, L., de Oliveira, M. G. X., de Oliveira, M. C. V., Gomes, C. R., et al. (2017). High-virulence CMY-2- and CTX-M-2-producing avian pathogenic Escherichia coli strains isolated from commercial turkeys. Diagn. Microbiol. Infect. Dis. 87, 64-67. doi: 10.1016/j.diagmicrobio.2016.10.001

Denny, J., Threlfall, J., Takkinen, J., Lofdahl, S., Westrell, T., Varela, C., et al. (2007). Multinational Salmonella Paratyphi B variant Java (Salmonella Java) outbreak, August - December 2007. Euro Surveill. 12:E071220.2. doi: 10.2807/esw.12.51.03332-en

Dierikx, C., van Essen-Zandbergen, A., Veldman, K., Smith, H., and Mevius, D. (2010). Increased detection of extended spectrum beta-lactamase producing Salmonella enterica and Escherichia coli isolates from poultry. Vet. Microbiol. 145, 273-278. doi: 10.1016/j.vetmic.2010.03.019

Donado-Godoy, P., Byrne, B. A., León, M., Castellanos, R., Vanegas, C., Coral, A., et al. (2015a). Prevalence, resistance patterns, and risk factors for antimicrobial resistance in bacteria from retail chicken meat in Colombia. J. Food Prot. 78, 751-759. doi: 10.4315/0362-028X.JFP-14-349

Donado-Godoy, P., Castellanos, R., León, M., Arevalo, A., Clavijo, V., Bernal, J., et al. (2015b). The establishment of the Colombian Integrated Program for 
Antimicrobial Resistance Surveillance (COIPARS): a pilot project on poultry farms, slaughterhouses and retail market. Zoonoses Public Health 62, 58-69. doi: $10.1111 /$ zph.12192

Donado-Godoy, P., Clavijo, V., León, M., Arevalo, A., Castellanos, R., Bernal, J., et al. (2014). Counts, serovars, and antimicrobial resistance phenotypes of Salmonella on raw chicken meat at retail in Colombia. J. Food Prot. 77, 227-235. doi: 10.4315/0362-028X.JFP-13-276

Donado-Godoy, P., Clavijo, V., León, M., Tafur, M. A., Gonzales, S., Hume, M., et al. (2012a). Prevalence of Salmonella on retail broiler chicken meat carcasses in Colombia. J. Food Prot. 75, 1134-1138. doi: 10.4315/0362-028X.JFP11-513

Donado-Godoy, P., Gardner, I., Byrne, B. A., León, M., Perez-Gutierrez, E., Ovalle, M. V., et al. (2012b). Prevalence, risk factors, and antimicrobial resistance profiles of salmonella from commercial broiler farms in two important poultry-producing regions of colombia. J. Food Prot. 75, 874-883. doi: 10.4315/0362-028X.JFP-11-458

Doublet, B., Praud, K., Nguyen-Ho-Bao, T., Argudín, M. A., Bertrand, S., Butaye, P., et al. (2014). Extended-spectrum $\beta$-lactamase-and ampc $\beta$-lactamaseproducing D-tartrate-positive Salmonella enterica serovar Paratyphi B from broilers and human patients in Belgium, 2008-10. J. Antimicrob. Chemother. 69, 1257-1264. doi: 10.1093/jac/dkt504

Dutil, L., Irwin, R., Finley, R., Ng, L. K., Avery, B., Boerlin, P., et al. (2010). Ceftiofur resistance in Salmonella enterica serovar Heidelberg from chicken meat and humans, Canada. Emerging Infect. Dis. 16, 48-54. doi: 10.3201/eid1601. 090729

Edgar, R. C. (2004). MUSCLE: a multiple sequence alignment method with reduced time and space complexity. BMC Bioinformatics 5:113. doi: 10.1186/1471-2105-5-113

European Food Safety Authority (EFSA) (2007). The community summary report on trends and sources of zoonoses, zoonotic agents, antimicrobial resistance and foodborne outbreaks in the european union in 2006. EFSA J. 1300, 1-7. doi: 10.2903/j.efsa.2007.130r

European Food Safety Authority (EFSA) and European Centre for Disease Prevention and Control (ECDC). (2016). The European Union summary report on trends and sources of zoonoses, zoonotic agents and food-borne outbreaks in 2015. EFSA J. 14:4634. doi: 10.2903/j.efsa.2016.4634

Foley, S. L., Nayak, R., Hanning, I. B., Johnson, T. J., Han, J., and Ricke, S. C. (2011). Population dynamics of Salmonella enterica serotypes in commercial egg and poultry production. Appl. Environ. Microbiol. 77, 4273-4279. doi: 10.1128/AEM.00598-11

Folster, J. P., Pecic, G., Bolcen, S., Theobald, L., Hise, K., and Carattoli, A. (2010). Characterization of extended-spectrum cephalosporin - resistant Salmonella enterica serovar heidelberg isolated from humans in the United States. Foodborne Pathog. Dis. 7, 181-187. doi: 10.1089/fpd.2009. 0376

Folster, J. P., Pecic, G., Singh, A., Duval, B., Rickert, R., Ayers, S., et al. (2012). Characterization of extended-spectrum cephalosporin-resistant Salmonella enterica serovar heidelberg isolated from food animals, retail meat, and humans in the United States 2009. Foodborne Pathog. Dis. 9, 638-645. doi: $10.1089 /$ fpd.2012.1130

Hendriksen, R. S., Vieira, A. R., Karlsmose, S., Lo Fo Wong, D. M. A., Jensen, A. B., Wegener, H. C., et al. (2011). Global monitoring of Salmonella serovar distribution from the world health organization global foodborne infections network country data bank: results of quality assured laboratories from 2001 to 2007. Foodborne Pathog. Dis. 8, 887-900. doi: 10.1089/fpd. 2010.0787

Hopkins, K. L., Deheer-Graham, A., and Threlfall, E. J. (2006). Novel plasmid-mediated CTX-M-8 subgroup extended-spectrum $\beta$-lactamase (CTXM-40) isolated in the UK. Int. J. Antimicrob. Agents 27, 570-572. doi: 10.1016/j.ijantimicag.2006.03.003

Jarquin, C., Alvarez, D., Morales, O., Morales, A. J., López, B., Donado, P., et al. (2015). Salmonella on raw poultry in retail markets in guatemala: levels, antibiotic susceptibility, and serovar distribution. J. Food Prot. 78, 1642-1650. doi: 10.4315/0362-028X.JFP-15-117

Jiang, X., Zhang, Z., Li, M., Zhou, D., Ruan, F., and Lu, Y. (2006). Detection of extended-spectrum $\beta$-lactamases in clinical isolates of Pseudomonas aeruginosa. Antimicrob. Agents Chemother. 50, 2990-2995. doi: 10.1128/AAC.01511-05
Larsen, M. V., Cosentino, S., Rasmussen, S., Friis, C., Hasman, H., Marvig, R. L., et al. (2012). Multilocus sequence typing of total-genome-sequenced bacteria. J. Clin. Microbiol. 50, 1355-1361. doi: 10.1128/JCM.06094-11

Liakopoulos, A., Geurts, Y., Dierikx, C. M., Brouwer, M. S. M., Kant, A., Wit, B., et al. (2016). Extended-spectrum cephalosporin- resistant Salmonella enterica serovar Heidelberg strains, the Netherlands. Emerg Infect. Dis. 22, 1257-1261. doi: 10.3201/eid2207.151377

Mevius, D. J., Dierikx, C. M., Veldman, K. T., Wit, B., van Pelt, W., and Heederik, D. (2015). Monitoring of Antimicrobial Resistance and Antibiotic Usage in Animals in the Netherlands in 2014 (MARAN). Available online at: https://www.wur.nl/upload_mm/e/0/d/104f4c0c-1126-4b64-b55b80bed717802a_NethmapMaran2015.pdf

Mughini-Gras, L., Enserink, R., Friesema, I., Heck, M., Van Duynhoven, Y., and Van Pelt, W. (2014). Risk factors for human salmonellosis originating from pigs, cattle, broiler chickens and egg laying hens: a combined case-control and source attribution analysis. PLoS ONE 9:e87933. doi: 10.1371/journal.pone.00 87933

Nadon, C., Van Walle, I., Gerner-Smidt, P., Campos, J., Chinen, I., ConcepcionAcevedo, J., et al. (2017). Pulsenet international: vision for the implementation of whole genome sequencing (WGS) for global foodborne disease surveillance. Eur. Surveill. 22:30544. doi: 10.2807/1560-7917.ES.2017.22.23. 30544

Paauw, A., Fluit, A. C., Verhoef, J., and Leverstein-Van Hall, M. A. (2006). Enterobacter cloacae outbreak and emergence of quinolone resistance gene in Dutch Hospital. Emerg. Infect. Dis. 12, 807-812. doi: 10.3201/eid1205. 050910

Parks, D. H., Imelfort, M., Skennerton, C. T., Hugenholtz, P., and Tyson, G. W. (2015). CheckM: assessing the quality of microbial genomes recovered from isolates, single cells, and metagenomes. Genome Res. 25, 1043-1055. doi: $10.1101 /$ gr.186072.114

Price, M. N., Dehal, P. S., and Arkin, A. P. (2010). FastTree 2 - Approximately maximum-likelihood trees for large alignments. PLoS ONE 5:e9490. doi: 10.1371 /journal.pone. 0009490

Public Health Agency of Canada (2016). Canadian Antimicrobial Resistance Surveillance System Report 2016. Available online at: https://www.canada. ca/en/public-health/services/publications/drugs-health-products/canadianantimicrobial-resistance-surveillance-system-report-2016.html

Rodríguez, C. E., Díaz-guevara, P., Moreno, J., Bautista, A., Montano, L., Realpe, M. E., et al. (2017). Laboratory surveillance of Salmonella enterica from human clinical cases in Colombia 2005 - 2011. Enferm. Infecc. Microbiol. Clin. 35, 417-425. doi: 10.1016/j.eimc.2016.02.023

Siguier, P. (2006). ISfinder: the reference centre for bacterial insertion sequences. Nucleic Acids Res. 34, D32-D36. doi: 10.1093/nar/gkj014

Smith, H., Bossers, A., Harders, F., Wu, G., Woodford, N., Schwarz, S., et al. (2015). Characterization of epidemic IncI1-I $\gamma$ plasmids harboring ambler class A and C genes in Escherichia coli and Salmonella enterica from animals and humans. Antimicrob. Agents Chemother. 59, 5357-5365. doi: 10.1128/AAC. 05006-14

Treangen, T. J., Ondov, B. D., Koren, S., and Phillippy, A. M. (2014). The Harvest suite for rapid core-genome alignment and visualization of thousands of intraspecific microbial genomes. Genome Biol. 15:524. doi: 10.1186/s13059-014-0524-x

United States Department of Agriculture (USDA) - Food Safety and Inspection Service (FSIS) (2013). Serotypes Profile of Salmonella Isolates from Meat and Poultry Products January 1998 through December 2013. Available online at: https://www.fsis.usda.gov/wps/wcm/connect/c7b5903c-8e8b-4f85$9 \mathrm{~b} 5 \mathrm{c}-12$ eaf $990 \mathrm{~d} 2 \mathrm{dd} /$ Salmonella-Serotype-Annual-2013.pdf?MOD=AJPERES van Pelt, W., van der Zee, H., Wannet, W. J., van de Giessen, A. W., Mevius, D. J., Bolder, N. M., et al. (2003). Explosive increase of Salmonella Java in poultry in the Netherlands: consequences for public health. Eur. Surveill. 8, 31-35. doi: 10.2807/esm.08.02.00398-en

Veldman, K. T., Mevius, D. J., Wit, B., van Pelt, W., and Heederik, D. (2016). Monitoring of Antimicrobial Resistance and Antibiotic Usage in Animals in the Netherlands in 2015 (MARAN). Available online at: https://www.wur.nl/ upload_mm/0/b/c/433ca2d5-c97f-4aa1-ad34-a45ad522df95_92416_008804_ NethmapMaran2016\$+\$TG2.pdf

Voets, G. M., Fluit, A. C., Scharringa, J., Cohen Stuart, J., and LeversteinVan Hall, M. A. (2011). A set of multiplex PCRs for genotypic 
detection of extended-spectrum $\beta$-lactamases, carbapenemases, plasmid-mediated AmpC $\beta$-lactamases and OXA $\beta$-lactamases. Int. J. Antimicrob. Agents 37, 356-359. doi: 10.1016/j.ijantimicag.2011. 01.005

Wagenaar, J. A., Hendriksen, R. S., and Carrique-Mas, J. (2013). Practical considerations of surveillance of Salmonella serovars other than Enteritidis and Typhimurium. Rev. Sci. Tech. 32, 509-519. doi: 10.20506/rst.32. 2.2244

Yoshida, C. E., Kruczkiewicz, P., Laing, C. R., Lingohr, E. J., Gannon, V. P. J., Nash, J. H. E., et al. (2016). The Salmonella In Silico Typing Resource (SISTR): an open web-accessible tool for rapidly typing and subtyping draft Salmonella genome assemblies. PLoS ONE 11:e0147101. doi: 10.1371/journal.pone.01 47101

Zankari, E., Hasman, H., Cosentino, S., Vestergaard, M., Rasmussen, S., Lund, O., et al. (2012). Identification of acquired antimicrobial resistance genes. J. Antimicrob. Chemother. 67, 2640-2644. doi: 10.1093/jac/ $\mathrm{dks} 261$

Conflict of Interest Statement: The authors declare that the research was conducted in the absence of any commercial or financial relationships that could be construed as a potential conflict of interest.

Copyright (c) 2018 Castellanos, van der Graaf-van Bloois, Donado-Godoy, León, Clavijo, Arévalo, Bernal, Mevius, Wagenaar, Zomer and Hordijk. This is an openaccess article distributed under the terms of the Creative Commons Attribution License (CC BY). The use, distribution or reproduction in other forums is permitted, provided the original author(s) and the copyright owner(s) are credited and that the original publication in this journal is cited, in accordance with accepted academic practice. No use, distribution or reproduction is permitted which does not comply with these terms. 